\title{
Performance on a Motor Control Test in an Asymptomatic Adolescent Population
}

\author{
Kari Lindegren, PT, DPT, OCS ${ }^{1}$, Kristin Bastian, PT, DPT, OCS ${ }^{2}$, Christopher Kovacs, PT, DPT, OCS, CSCS ${ }^{2}$ Robyn \\ McHugh, PT, DPT, OCS, CSCS ${ }^{2}$ Catherine Quatman-Yates, PT, DPT, PhD ${ }^{3}$, Mark Paterno, PT, PhD, MBA, SCS, ATC \\ 1 Division of Pediatric Rehabilitation Medicine, Children's Hospital Los Angeles, 2 Division of Occupational and Physical Therapy, Cincinnati Children's \\ Hospital Medical Center; Division of Sports Medicine, Cincinnati Children's Hospital Medical Center, ${ }^{3}$ Department of Physical Therapy, School of \\ Health and Rehabilitation Sciences, College of Medicine, Sports Medicine Research Institute, The Ohio State University, ${ }^{4}$ Division of Occupational and \\ Physical Therapy, Cincinnati Children's Hospital Medical Center; Division of Sports Medicine, Cincinnati Children's Hospital Medical Center; \\ Department of Pediatrics, University of Cincinnati College of Medicine \\ Keywords: adolescent, core stability, motor control, low back pain, abdominal muscles, movement system
}

https://doi.org/10.26603/001c.31042

\section{International Journal of Sports Physical Therapy}

Vol. 17, Issue 2, 2022

\section{Background}

Low back pain is a condition present during both adulthood and adolescence. Adolescents with low back pain may benefit from treatment focused on improving abdominal muscle performance and motor control. The supine double leg lowering test (SDLLT) may be a reliable measure to assess core stability in adults, but adolescent performance on the SDLLT has not yet been established in the literature.

\section{Purpose}

To examine performance on the SDLLT in healthy adolescents ages 13 to 18 years and describe influences of gender, age, body mass index, and participation in sport.

\section{Study Design}

Cross-Sectional Study

\section{Methods}

Four licensed physical therapists administered the SDLLT with a Stabilizer pressure biofeedback cuff and inclinometer in 90 adolescents without low back pain (females $=41$, males $=49$ ) from three schools in a mid-western metropolitan area. Descriptive statistics, independent sample t-tests, two-way analysis of variance, and Pearson correlation coefficients were utilized to analyze the data.

\section{Results}

Average SDLLT score was 72.36 +/- 12.54 degrees. A significant difference between SDLLT score was present between genders with males performing better than females. No interactions between performance and involvement in sport were demonstrated.

\section{Conclusions}

Female and male adolescents appear to perform differently on the SDLLT with a stabilizer and appear to perform worse than scores recorded for adults. The SDLLT may be used to measure motor control in adolescents, but clinicians should utilize age-appropriate data for clinical decision making.

\section{Levels of Evidence}

Level 2c

\footnotetext{
a Corresponding Author:

Kari Lindegren, PT, DPT, OCS

Children's Hospital Los Angeles, 4650 Sunset Blvd., Mailstop \#56

Los Angeles, CA 90027.

Email: klindegren@chla.usc.edu
} 


\section{INTRODUCTION}

Low back pain (LBP) is no longer a condition that begins in middle age, with recent literature suggesting increased frequency of back pain in individuals under the age of 20 in both athletes and non-athletes. ${ }^{1-5}$ The increasing prevalence of adolescent LBP supports the need to optimize care for this population. In order to improve the care for this population, appropriate measures must be utilized for examination and assessment.

Previous authors have proposed a subgroup of adult patients benefiting from motor control exercises in the treatment of LBP. ${ }^{6-8}$ The core includes structures of the spine, hips, and pelvis and consists of both local and global musculature at the trunk and pelvis. ${ }^{9,10}$ Local musculature includes muscles with an insertion or origin at the vertebrae and include the transversus abdominis and multifidi. ${ }^{10}$ Global musculature includes the muscles that "transfer load between the thoracic cage and the pelvis" and include muscles such as the external obliques, internal obliques, rectus abdominis, erector spinae, and psoas. ${ }^{10(\mathrm{p} 20)}$ These muscles may be responsible for the skill acquisition of dissociating or coordinating lumbar spine movements from adjacent regions. ${ }^{8}$ While researchers have slight variations in their definition of the core, greater discrepancy exists in defining core stability. Core stability may be achieved through the interdependence of the passive ligamentous subsystem, active musculoskeletal subsystem, and neural feedback subsystem to match various demands due to changes in posture and load and may include proprioception, strength, power, and endurance. ${ }^{11,12}$

A clinical prediction rule exists for this subgroup of patients benefiting from core stabilization in the treatment of LBP, and recent findings suggest children and adolescents with LBP present with findings that often classify them as potentially benefiting from stabilization interventions. $6,7,13$ It is likely that adolescent patients present with deficits in core stability, however, no gold standard for measuring this impairment currently exists in the literature. ${ }^{11}$ One tool used to assess lumbopelvic motor control is the supine double leg lowering test (SDLLT), and previous authors have suggested that it may be a reliable measure of lumbopelvic motor control in adults. ${ }^{14-18}$

The SDLLT was originally described as an abdominal muscle strength measurement, but based off dynamometry studies, Ladeira et al. suggested that the test is more likely exploring pelvic tilt motor control required to maintain lumbar functional stability versus true abdominal strength.. 14,17,19,20 However, electromyographic (EMG) studies have reported moderate to high levels of abdominal muscle activation with the SDLLT, specifically the rectus abdominis, external obliques, internal obliques, and transversus abdominis. The results of these studies also indicate that the SDLTT does not isolate the lower abdominals, but rather requires high levels of abdominal co-contraction. ${ }^{15,21,22}$ Therefore, the muscle activity related to the SDLLT may be more indicative of assessing lumbopelvic motor control versus strength. ${ }^{11,14,17,21}$ However, motor control has recently been defined as "the way in which the nervous system controls posture and movement to perform a given task" through motor, sensory and integrative processes. ${ }^{23(p 380)}$ It is possible the SDLLT assesses the ability of an individual to control sagittal plane lumbar spine motion and manage the associated internal lumbar extension moment produced while performing the SDLLT. It is therefore suggested the SDLLT may be a more appropriate test of an individual's motor control rather than core stability.

Performance on the SDLLT in young adult and adult populations are reported in the literature but has not been investigated in the adolescent population. ${ }^{11,14,19,22}$ It is therefore imperative that performance on the SDLLT is examined in this population in order to allow clinicians to reliably assess deficits and improvements in motor control in order to improve clinical examination, evaluation, and treatment of low back pain in this population. The purpose of this study was to examine performance on the SDLLT in healthy adolescents ages 13 to 18 years and describe influences of gender, age, body mass index, and participation in sport.

\section{METHODS}

\section{PARTICIPANTS}

Adolescents between the ages of 13-18 years were recruited from physical education classes at three schools in a midwestern metropolitan area between April 2017 and April 2018. Each parent or 18-year-old participant signed a written informed consent and adolescents under the age of 18 years signed a written informed assent. Exclusion criteria for this study included: (1) being younger than 13 years and/ or older than 18 years, (2) a current diagnosis or previous history of low back pain, scoliosis, spondylolisthesis, and/ or spondylolysis, (3) current musculoskeletal complaints in the upper or lower extremity including pain, radiating symptoms, or impairment, (4) previous history of spine, upper extremity, or lower extremity surgery, or (5) pain-related to acute fracture, tumor, infection, or systemic illness. This study had approval from the Cincinnati Children's Hospital Medical Center Institutional Review Board.

\section{PARTICIPANT CHARACTERISTICS DATA}

Participants self-reported age in years, gender, height, weight, and athletic participation prior to performing the SDLLT. Body mass index (BMI) was calculated utilizing participant's self-reported height and weight and then categorized based on recommendations from the Centers for Disease Control and Prevention (CDC). ${ }^{24}$ Athletic participation was first categorized by participation in any activity and then further categorized by type of organized sport.

\section{PROCEDURE}

All adolescents were tested during their physical education class period. Four licensed physical therapists trained in performing and measuring the SDLLT using a standardized protocol administered the test to participants. (Figure 1). ${ }^{11,16}$ A stabilizer pressure biofeedback unit (Chattanooga Group Inc, Vista, CA) is a small pressure biofeedback device typically used by physical therapists during examination and treatment of patients with low back pain. The stabilizer 
was used to determine when to terminate the SDLLT (Figure 2). A single inclinometer (Baseline AccuAngle Goniometer, Japan) was used to record the hip flexion angle of the SDLLT. One examiner was blinded from the inclinometer measurement in order to eliminate the potential for intrarater bias while measuring.

After providing verbal consent, each participant took off their shoes and lay supine on a standard, portable treatment table with arms across their chest. The stabilizer was placed under the participant's lumbar spine and their legs were passively elevated to 90 degrees of hip flexion from the horizontal (Figure 1A). To account for hamstring tightness, 5-10 degrees of knee flexion was considered acceptable. They were instructed to keep their legs at 90 degrees without assistance while the stabilizer was inflated to 40 $\mathrm{mmHg}$. The following statement was given to each participant prior to completing the test: "Press your back down into the stabilizer while keeping your legs straight. Keep pressing your back into the stabilizer as you slowly lower both your legs towards the table keeping your knees straight. Don't let your back come up from the stabilizer under you."

The test was ended when the pressure reading from the stabilizer fell below $30 \mathrm{mmHg}$. At this point, Examiner one passively supported the participant's legs while Examiner two utilized the single inclinometer to record the angle of their legs from the horizontal. When the SDLLT is ended, a hip flexion angle closer to 0 degrees of hip flexion indicates better performance whereas a hip flexion angle closer to 90 degrees of hip flexion indicates worse performance. (Figures 1B and 1C). Each participant received one practice trial with the stabilizer in place followed by a one-minute rest break. They then performed two recorded trials of the SDLLT with another one-minute rest break in between the trials. The above protocol was determined based on previous research published in the literature. ${ }^{2,3}$ A previous pilot study using the same protocol was used to determine reliability and found the SDLLT to have high intra-rater reliability $(0.885, \mathrm{p}<0.001)$ as well as high inter-rater reliability $(0.832, \mathrm{p}<0.001) .^{18}$

\section{DATA ANALYSIS}

SDLLT scores were recorded in Microsoft Excel 2013 (Microsoft Corporation, Redmond, WA). Data were analyzed using Microsoft Excel 2013 and IBM SPSS Statistics Version 25 (IBM Corporation, Armonk, NY). Descriptive statistics were utilized to characterize the sample with regard to: age in years, gender, body mass index, height, weight, athletic participation, and performance on the SDLLT. Average SDLLT score was calculated from the two trials and categorized by gender, age in years, athletic participation, height, weight, and BMI. Independent sample t-tests and two-way analysis of variance (ANOVA) were utilized to determine whether significant differences were present between subgroups with a priori significance set to 0.05 . Pearson correlation coefficients were analyzed to further determine a correlation between age and SDLLT score, age and BMI, and BMI and SDLLT score.

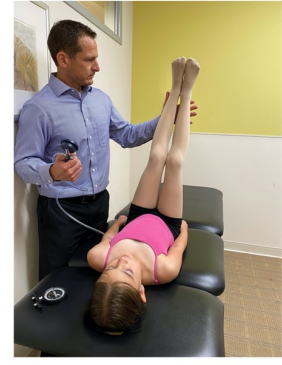

A
B

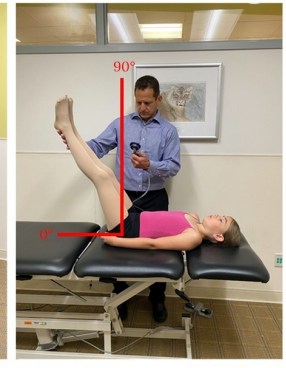

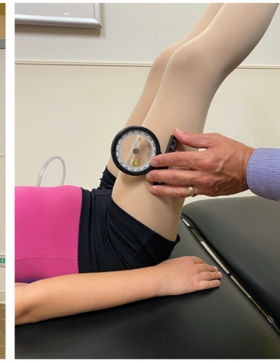

C

\section{Figure 1. Images of the SDLLT examination protocol}

(A) Starting position of the SDLLT. (B) Ending position of the SDLLT. (C) Measurement of the SDLLT utilizing an inclinometer.

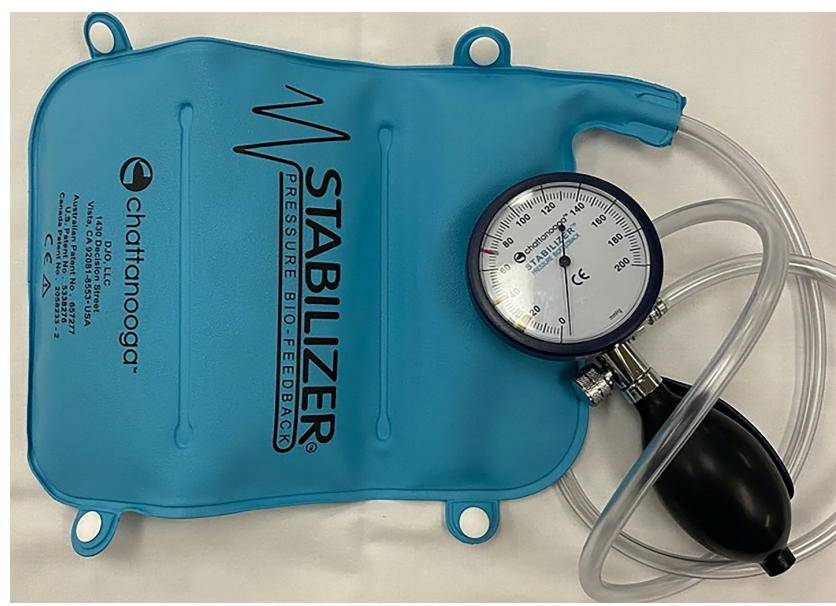

Figure 2. Image of the stabilizer pressure biofeedback unit

\section{RESULTS}

\section{SAMPLE DESCRIPTION}

A total of 142 adolescents volunteered to participate in this study and 90 met eligibility criteria (41 females, 49 males). Additional information on participant selection is presented in Figure 3 . The mean age was 15.88 years +/- 1.31 years (range 13.00-18.00 years). The average height of participants was $67.93+/-4.12$ inches (range 59.00-77.00 inches) and the average weight was $148.12+/-37.41$ pounds (range 70.00-334.60 pounds). The average BMI of all participants was $22.45+/-5.01$ (range 8.52-49.41). Out of the 90 participants, height information was not available for two, weight information was not available for one, and BMI information was not available for two. Additional sample characteristic information is presented in Table 1.

Based on CDC classifications for BMI, 10\% of the sample in this study was underweight, $72 \%$ were a healthy weight, $11 \%$ were overweight, and $4 \%$ were obese. ${ }^{24}$ Of all participants included in the study, 56 reported they were involved in a type of organized sport whereas 34 reported they were not involved in any type of organized sport. Thirty-six participants were involved in a single sport and 20 participants were involved in two or more sports. Soccer, track and field, 
Table 1. Sample Demographics

\begin{tabular}{|c|l|l|l|}
\hline Characteristic & Females $(\mathbf{n}=41)$ & Males $(\mathbf{n}=49)$ & Total $(\mathrm{n}=90)$ \\
\hline Age (years) & & & \\
\hline Mean & 15.51 & 16.18 & 15.88 \\
\hline Standard Deviation (range) & $1.19(13-18)$ & $1.33(14-18)$ & $1.31(13-18)$ \\
\hline Height (inches) & & & 67.93 \\
\hline Mean & 64.95 & 70.43 & $4.12(59-77)$ \\
\hline Standard Deviation (range) & $3.05(59-76)$ & $3.12(64-77)$ & 148.12 \\
\hline Weight (pounds) & & & $37.41(70-334)$ \\
\hline Mean & 128.33 & 164.42 & \\
\hline Standard Deviation (range) & $24.06(70-200)$ & $38.72(111-334.6)$ & 22.45 \\
\hline BMI & & & $5.01(8.52-49.41)$ \\
\hline Mean & 21.47 & 23.28 & \\
\hline Standard Deviation (range) & $3.90(8.52-32.28)$ & $5.68(17.70-49.41)$ & \\
\hline
\end{tabular}

and football were the most commonly represented sports with each having greater than or equal to 10 participants.

\section{SDLLT PERFORMANCE}

Healthy adolescents without pain scored an average of $72.36+/-12.54$ degrees on the SDLLT. SDLLT scores for the entire group and for males and females are presented in Figure 4 .

There was a significant difference in SDLLT score between females (76.99+/- 6.98 degrees) and males (68.49+/14.74 degrees) suggesting females and males perform differently on the SDLLT $(p=0.001)$. There was no significant difference between participants who were involved in organized sports and participants who were not involved in organized sports $(\mathrm{p}=0.849)$.

A two-way analysis of variance was used to assess interaction between gender, participation in an organized sport, and SDLLT score. No interaction was found between participation in an organized sport and SDLLT score $(\mathrm{p}=0.849)$ or between gender and participation in organized sport $(\mathrm{p}=0.587)$.

Correlations were assessed between age and SDLLT score, BMI and age, and BMI and SDLLT score. There was a significant weak negative correlation between age and SDLLT score $(\mathrm{r}=-0.218, \mathrm{p}=0.04)$ and a significant weak positive correlation between BMI and age $(r=0.229, p=0.03)$. No significant correlation was found between BMI and performance on the SDLLT $(r=-0.113, p=0.30)$.

\section{DISCUSSION}

The purpose of this study was to describe SDLLT performance as measured using a stabilizer in healthy adolescents without pain using a previously established standardized protocol. Previous authors have suggested that female and male adults perform differently on the SDLLT and results of this study demonstrate a difference in adolescent performance on the SDLLT between gender, suggesting that ado-

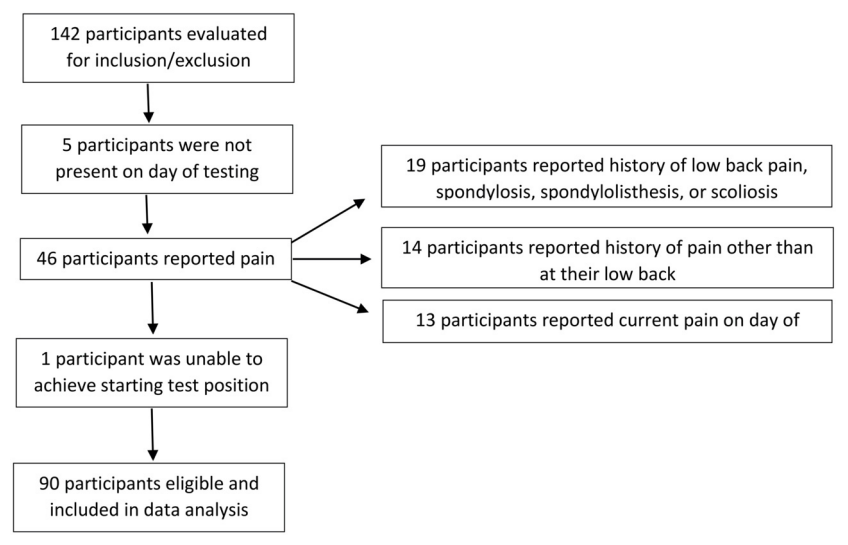

Figure 3. Participant Eligibility

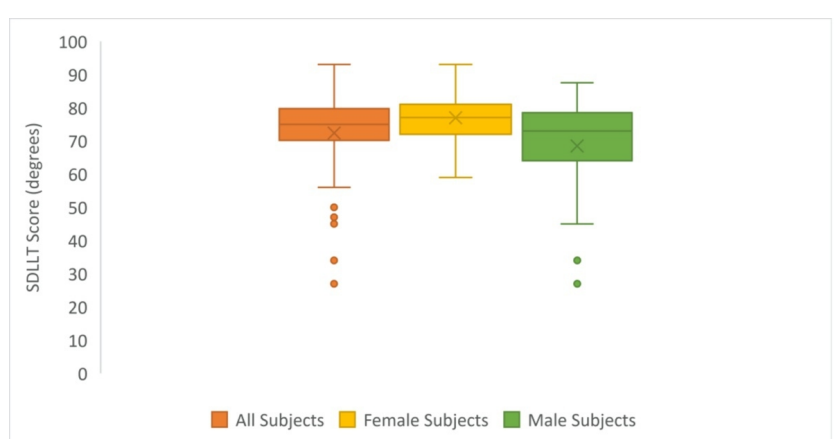

Figure 4. Performance on the SDLLT for healthy adolescents ages $13-18$ years

lescent females and males also perform differently on the SDLLT. ${ }^{11,14,22}$ As age increased, BMI also increased with no relationship between BMI and performance on the SDLLT.

Compared to previously published studies, the results of this study indicate adolescents may not perform as well as 
adults on the SDLLT. ${ }^{11,14,19,22}$ Literature assessing adults over 18 years of age suggests normal scores for the SDLLT to be between 15 degrees and 55 degrees. Youdas et al. ${ }^{25}$ described normal scores on the SDLLT in adults ages 40 to 69 years of 49.6 degrees for females and 39.0 degrees for males, Krause et al. ${ }^{14}$ described normal on the SDLLT in adults ages 18 to 29 years as 36.9 degrees for females and 15.4 degrees for males, and Sharrock et al. ${ }^{11}$ described normal values in adults ages 18 to 22 years of 54.8 degrees for females and 47.4 degrees for males. In contrast, adolescents ages 13 to 18 years in this study performed an average of 77.0 degrees females and an average of 68.5 degrees for males. Poorer performance on the SDLLT in this study as compared to previously published studies may be due to differences in maturation, cognition, and neuromuscular control in the adolescent population compared to adults. Future directions should further evaluate any differences in SDLLT score between specific ages due to potential differences in maturation and peak height velocity which can lead to variable performances.

Differences in SDLLT performance may also be due to protocol. Kendall first described the SDLLT and used a hand under the lumbar spine to determine lumbopelvic motion and when to decide the end of the test. ${ }^{19}$ Krause et al. ${ }^{14}$ also used an examiner's hand to assess for an increase in lumbar lordosis indicating the end of the test. The protocol in this study utilized a stabilizer similar to that described by Sharrock et al. ${ }^{11}$ to determine the end of the test. The stabilizer is likely a more objective measure and more sensitive and reliable than an examiner's hand in detecting changes in lumbar lordosis, which may contribute to the poorer scores on the SDLLT in this study compared to previous studies. The large number of participants who performed between 80 and 90 degrees on the SDLLT may indicate excessive sensitivity and responsiveness of the stabilizer to movement in the pelvis/lumbar spine, based on this study's protocol. This study also utilized a single inclinometer instead of a goniometer which may contribute to differences in the measurement of the performance in this study compared to previous studies.

There were no significant differences between participants who were involved in sports and those who did not. Previous work has reported differences in prevalence of low back pain in adolescent athletes participating in combat sports or sports requiring repetitive translation or rotation forces. ${ }^{2}$ With increasing evidence emerging on the relationship of motor control to athletic performance, it is possible that athletes participating in sports requiring skills similar to the SDLLT or demanding repetitive rotational forces and high impact may perform differently on the SDLLT compared to other athletes. Further, adolescent athletes may require greater amounts of motor control for sports performance and the SDLLT may assist clinicians in assessing motor control. Future studies investigating performance by sport may further clarify the potential impact of specific sports on SDLLT performance.

Adolescents present with unique characteristics related to physical and cognitive development that contribute to differences in movement compared to adults. Despite differences between adults and adolescents, clinicians often utilize clinical measures studied in adults with established normative values based on adult performance in the pediatric and adolescent population. As previously mentioned, studies on the prevalence of low back pain suggest differences exist in adolescents compared to adults. ${ }^{2}$ While repetitive microtrauma can lead to low back pain within both the adult and adolescent populations, low back pain in adolescents may also be due to insufficiency within the muscle-tendon complex due to decreased neuromuscular control and impaired posture. ${ }^{2}$ Due to the demands of the SDLLT, it is possible that the SDLLT requires both strength and neuromuscular control to dissociate hip extension from lumbar extension and anterior pelvic tilt. With the mean SDLLT score of adolescents ages 13 to 18 years appearing worse than values seen in adults, it is important that clinicians utilize values on performance specific to the population they are treating in order to determine whether an impairment in motor control is present, assess meaningful changes in performance, and set reasonable and achievable goals. While no reference values currently exist for SDLLT performance in the adolescent population, the findings from this study may assist clinicians in determining a patient's progress as they return to their prior level of function or optimize their current level of function.

\section{LIMITATIONS}

Several limitations may exist in this study. First, the relatively small sample size of this study may limit the generalizability of the findings to the general population. Second, this study did not account for leg length which may change the findings of this study. Although it does not appear that weight, height, and BMI influence performance on the SDLLT, the length of the lower extremities may affect the amount of muscle recruitment and motor control required to perform the SDLLT. Third, while this study allowed up to 10 degrees of knee flexion to account for hamstring tightness, hamstring flexibility was not formally assessed and excessive hamstring tightness may impact the generalizability of the results. In addition, decreased hamstring length in the starting testing position may cause a passive posterior pelvic tilt which may have affected performance and clinicians should be mindful of this when utilizing the SDLLT. Fourth, this study relied on verbal reports of height and weight which may also alter the relationship between these variables and performance on the SDLLT. Fifth, while students were pulled from physical education class randomly and at different times during class to perform the SDLLT, fatigue from physical education class was not controlled for. Lastly, for several participants, performance on the second trial was improved compared to the first trial. This may be due to a learning effect on the SDLLT or it is possible that the SDLLT is a more novel task to adolescents compared to adults. This may explain differences in performance between adolescents and adults as well as the improved performance between trials in some participants. It is also possible that adolescents utilized different movement strategies to maintain pressure on the stabilizer.

Additional work is needed to further examine the reliability and validity of the SDLLT in the adolescent population. Future directions may include establishing normative values for performance on the SDLLT in a larger sample size 
utilizing the standardized protocol described in this study. It is recommended that future studies account for leg length in order to determine whether the length of the lower extremities influence performance on the SDLLT. Due to the large number of participants performing between 80 and 90 degrees on the SDLLT, future research should consider utilizing a different pressure reading on the stabilizer to determine the end of the SDLLT. Future work should also focus on comparing performance on other trunk motor control tests between adults and adolescents in order to determine whether a trend in poorer performance is present in other tests besides the SDLLT. It is important that future work also examine performance on the SDLLT in relation to maturation status and/or cognition since these factors may have affected performance on the SDLLT. Lastly, future studies should compare performance on the SDLLT in adolescents with low back pain to adolescents without low back pain. In this study, none of the participants reported back pain while administering the SDLLT. However, the onset of pain during the SDLLT may impact performance on this test. Clinically, the SDLLT is often terminated or modified with the onset of back pain and performance may be reported as 90 degrees with pain in the starting position.

\section{CONCLUSION}

To the author's knowledge, this is the first study to examine performance on the SDLLT utilizing a standardized methodology involving a stabilizer in adolescents ages 13 to 18 years. The results of this study indicate that female and male adolescents appear to perform differently on the SDLLT, but it does not appear that there is a relationship between SDLLT score and BMI. Performance on the SDLLT in adolescents also appears to be worse than in performance by adults reported in previous studies. The SDLLT is simple to use in a clinic setting, requires minimal equipment, and seems to have minimal to no adverse effects. The SDLLT may be a useful measure for physical therapists to assess motor control in adolescents and compare performance to that of healthy adolescents without pain or impairment to assist in clinical decision making.

\section{CONFLICTS OF INTEREST}

The authors report no conflicts of interest.

Submitted: January 12, 2021 CST, Accepted: October 13, 2021 CST 


\section{REFERENCES}

1. Swain MS, Henschke N, Kamper SJ, Gobina I, Ottová-Jordan V, Maher CG. An international survey of pain in adolescents. BMC Public Health. 2014;14(1). doi:10.1186/1471-2458-14-447

2. Müller J, Müller S, Stoll J, Fröhlich K, Otto C, Mayer F. Back pain prevalence in adolescent athletes. Scand J Med Sci Sports. 2017;27(4):448-454. doi:10.1111/sm s.12664

3. Schmidt CP, Zwingenberger S, Walther A, et al. Prevalence of low back pain in adolescent athletes an epidemiological investigation. Int J Sports Med. 2014;35(8):684-689. doi:10.1055/s-0033-1358731

4. King S, Chambers CT, Huguet A, et al. The epidemiology of chronic pain in children and adolescents revisited: a systematic review. Pain. 2011;152:2729-2738. doi:10.1016/j.pain.2011.07.016

5. Jeffries LJ, Milanese SF, Grimmer-Somers KA. Epidemiology of adolescent spinal pain: a systematic overview of the research literature. Spine. 2007;32:2630-2637. doi:10.1097/brs.0b013e318158d7 $\mathrm{Ob}$

6. Delitto A, George SZ, Van Dillen LR, et al. Low back pain. J Orthop Sports Phys Ther. 2012;42(4):A1-57. do $\mathrm{i}: 10.2519 /$ jospt.2012.42.4.a1

7. Fritz JM, Cleland JA, Childs JD. Subgrouping patients with low back pain: evolution of a classification approach to physical therapy. J Orthop Sports Phys Ther. 2007;37(6):290-302. doi:10.2519/jos pt.2007.2498

8. Alrwaily M, Timko M, Schneider M, et al. Treatment-based classification system for patients with low back pain: the movement control approach. Phys Ther. 2017;97(12):1147-1157. doi:10.1093/ptj/pz $\underline{\mathrm{x} 087}$

9. Kibler WB, Press J, Sciascia A. The role of core stability in athletic function. Sports Med. 2006;36(3):189-198. doi:10.2165/00007256-20063603 $\underline{0-00001}$

10. Bergmark A. Stability of the lumbar spine. A study in mechanical engineering. Acta Orthop Scand Suppl. 1988;230:1-54.

11. Sharrock C, Cropper J, Mostad J, et al. A pilot study of core stability and athletic performance: is there a relationship? Int J Sports Phys Ther. $2011 ; 6(2): 63-74$.
12. Panjabi MM. The stabilizing system of the spine. Part I. Function, dysfunction, adaptation, and enhancement. J Spinal Disord. 1992;5(4):383-389. do i:10.1097/00002517-199212000-00001

13. Wall CA, Allen MD, McHugh RB, et al. Subgrouping children and adolescents with low back pain: is the treatment-based classification system useful? J Orthop Sports Phys Ther. 2015;45(1):A74-A151. doi:10.2519/jospt.2015.45.1.a7 4

14. Krause DA, Youdas JW, Hollman JH, Smith J. Abdominal muscle performance as measured by the double leg-lowering test. Arch Phys Med Rehabil. 2005;86(7):1345-1348. doi:10.1016/j.apmr.2004.12.02 $\underline{0}$

15. Shields RK, Heiss DG. An electromyographic comparison of abdominal muscle synergies during curl and double straight leg lowering exercises with control of the pelvic position. Spine (Phila Pa 1976). 1997;22(16):1873-1879. doi:10.1097/00007632-19970 8150-00012

16. Lanning CL, Uhl TL, Ingram CL, et al. Baseline values of trunk endurance and hip strength in collegiate athletes. J Athl Train. 2006;41(4):427.

17. Ladeira CE, Hess LW, Galin BM, et al. Validation of an abdominal muscle strength test with dynamometry. J Strength Cond Res. 2005;19(4):925-930.

18. Lindegren K, Bastian K, McHugh R, et al. Interrater and intrarater reliability of a core stability performance test. J Orthop Sports Phys Ther. 2017;47(1):A58-A161.

19. Kendall FP, Kendall FP. Muscles: Testing and Function with Posture and Pain. 5th ed. Lippincott Williams \& Wilkins; 2005.

20. Zannotti CM, Bohannon RW, Tiberio D, Dewberry MJ, Murray R. Kinematics of the double-leg-lowering test for abdominal muscle strength. J Orthop Sports Phys Ther. 2002;32(9):432-436. doi:10.2519/jospt.200 2.32.9.432

21. Haladay DE, Denegar CR, Miller SJ, Challis J. Electromyographic and kinetic analysis of two abdominal muscle performance tests. Physiother Theory Pract. 2015;31(8):587-593. doi:10.3109/095939 $\underline{85.2015 .1062945}$ 
22. Haladay DE, Miller SJ, Challis JH, Denegar CR. Responsiveness of the double limb lowering test and lower abdominal muscle progression to core stabilization exercise programs in healthy adults: a pilot study. J Strength Cond Res.

2014;28(7):1920-1927. doi:10.1519/jsc.000000000000 0336

23. van Dieën JH, Reeves NP, Kawchuk G, van Dillen LR, Hodges PW. Analysis of motor control in patients with low back pain: a key to personalized care? J Orthop Sports Phys Ther. 2019;49(6):380-388. doi:10.2 519/jospt.2019.7916
24. About Adult BMI. Centers for Disease Control and Prevention; 2017. Accessed January 30, 2020. http s://www.cdc.gov/healthyweight/assessing/bmi/adul t bmi/index.html

25. Youdas JW, Garrett TR, Harmsen S, Suman VJ, Carey JR. Lumbar lordosis and pelvic inclination of asymptomatic adults. Phys Ther. 1996;76(10):1066-1081. doi:10.1093/ptj/76.10.1066 